\title{
TEMPORARY CUTANEOUS URETEROSTOMY IN THE MANAGEMENT OF ADVANCED CONGENITAL URINARY OBSTRUCTION*
}

BY

\author{
J. H. JOHNSTON \\ From Alder Hey Children's Hospital, Liverpool
}

The most extreme effects of chronic urinary obstruction are seen in the child who has suffered a severe lower tract obstruction during foetal existence. In such cases the renal tract is dilated, sometimes dysplastic and often decompensated, so that urinary stasis commonly persists after the removal of the original obstruction. One has to deal with a urinary system which has in many instances never been normal and which, in most, is quite incapable of approaching normality. Some cases have insufficient renal tissue to maintain life, but many, if given the chance, have the capacity for considerable improvement in the function both of the urinary tract musculature and of the renal parenchyma. The great danger to the patient with a low renal reserve and urinary stasis is complicating infection; this is often present at the time of diagnosis or is quickly introduced by instrumentation or by operation, and if free urinary drainage is not established a rapidly fatal pyelonephritis may very readily develop.

Rickham (1962) has described our unhappy experience in Liverpool, particularly in infants, with suprapubic cystostomy in the management of advanced lower urinary obstruction. These poor results are due partly to the presence of the tube which perpetuates and aggravates infection, and partly, I suspect, to hypotonic ureters finding evacuation even more difficult when a very hypertrophied bladder is empty and contracted. Tube nephrostomy has also, in my experience, been unsatisfactory with the hydronephrotic kidney; although adequate drainage is obtained, there is again the great disadvantage of the indwelling catheter.

The ideal method of prolonged decompression in advanced urinary obstruction should drain the upper tract directly without the use of tubes. A technique that fulfils these criteria, and of which

\footnotetext{
* A paper read at a meeting of the British Association of Paediatric Surgeons in London, September 1962.
}

I have had experience in 10 patients with severely damaged urinary tracts from a variety of causes, is that of temporary cutaneous ureterostomy with later restoration of the normal urinary route after the obstruction has been removed. Six of the patients were infant boys with urethral valves; four of them had bilateral ureterostomy and two unilateral since these each had only one functioning kidney. One of these children died of staphylococcal pneumonia; his renal function was extremely poor, the para-aminohippuric acid (PAH) clearance being only $2.5 \%$ of normal. An infant girl with bilateral ectopic ureteroceles obstructing all four duplicated ureters and with only one double kidney functioning had a unilateral double ureterostomy: a girl of 21 months had a unilateral ureterostomy for an obstructive megaureter affecting a solitary, very poorly functioning kidney: a $3 \frac{1}{2}$-year-old boy had bilateral ureterostomy for a congenital obstruction of the penile urethra, and an infant boy had bilateral ureterostomy for an undiagnosed temporary urinary obstruction. In 13 instances in seven patients the ureterostomy was of the loop type described by Stephens (F. D. Stephens, 1960, personal communication), the ureter being exposed extraperitoneally through a small high gridiron incision, mobilized, brought in continuity to the surface and opened on its anterior aspect (Fig. 1). The elongated ureter that results from prenatal urinary obstruction can usually be exteriorized easily without tension and without endangering its blood supply. In three instances in three patients the ureterostomy was of the terminal type, the ureter being divided near the bladder and the proximal end brought to the surface. In two of these cases the obstruction lay at the ureterovesical junction. The ureter reaches the skin surface much more readily as a terminal stoma, but closure of this type of ureterostomy requires reimplantation of the ureter to the bladder, and when this would not be required in any case for the relief of the original obstruction or for the cure of 


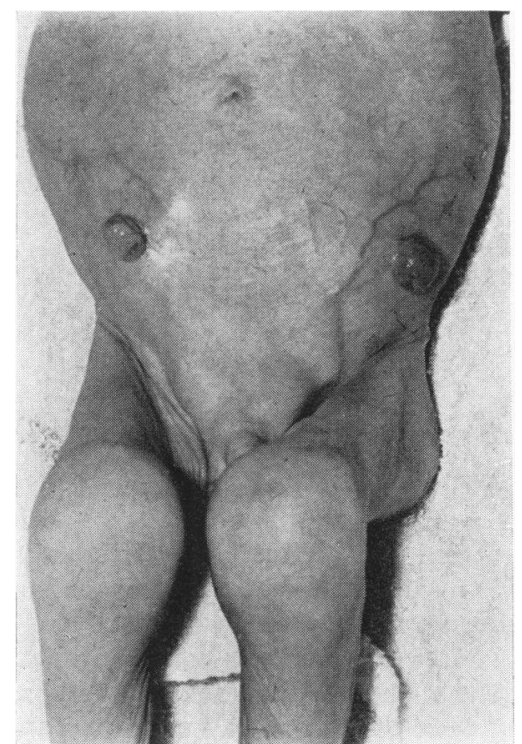

FIG. 1.-Bilateral loop ureterostomies in infant boy with urethral valves.

vesico-ureteric reflux, the loop ureterostomy is obviously preferable. With the exception of one patient who will be described later, local complications with either type of ureterostomy were minimal. Stomal stenosis was not seen in any case; some

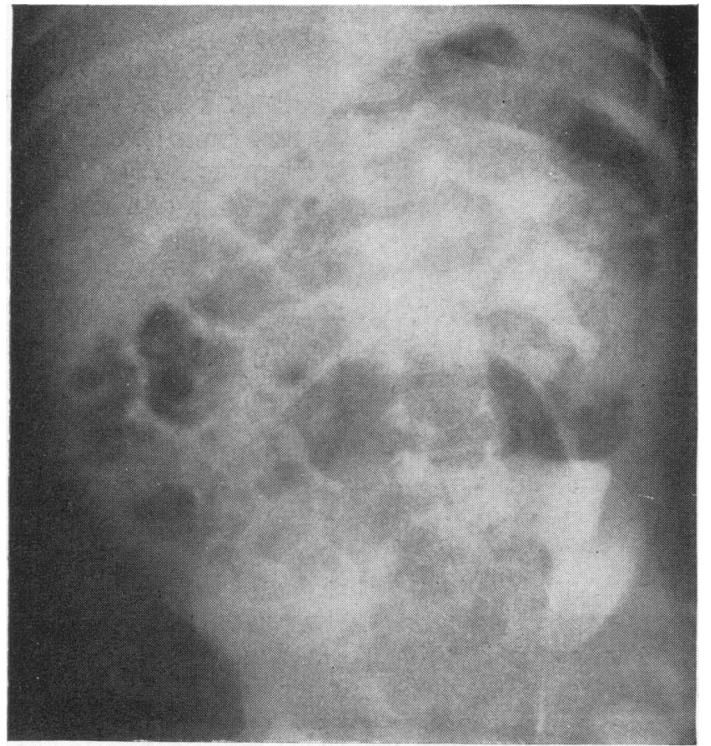

FIG. 2.-Intravenous pyelogram of Case 1 before resection of urethral valves and bilateral ureterostomy closure. Although the right ureterostomy drained urine the kidney did not concentrate sufficiently to appear on the radiograph; left hydronephrosis and hydroureter to site of ureterostomy. degree of ureteric prolapse occurred with one loop ureterostomy.

Ureterostomy may not immediately allow adequate emptying of a severely dilated upper renal tract. The kidney and ureter are, however, easily evacuated by bimanual compression and massage of the loin, and this manoeuvre should be carried out at frequent intervals by the nursing staff to prevent urinary accumulation. As the tone of the pelvic and ureteric muscle recovers the residual urine diminishes and one can appreciate improvement in ureteric peristalsis and emptying by repeated image intensifier observation.

The renal function in the children in this series has shown, in varying degrees of severity, the tubule defects of chronic back pressure, the most obvious features being uraemia, acidosis and obligatory polyuria with hyposthenuria. In five kidneys in three patients clearances of urea, creatinine and PAH were estimated shortly after the performance of ureterostomy and again, several weeks later, before its closure. In none did the tests show any significant change so that there is no objective proof that any recovery of renal function occurred in these cases as a result of drainage. That recovery can and does occur, however, is implied by the clinical improvement that ureterostomy has produced in all patients. This was particularly striking in three children in whom ureterostomy was performed as a secondary procedure when other methods of drainage had failed to be effective, and they were extremely ill. In most cases clinical recovery appeared shortly after ureterostomy drainage was instituted, but in one it was considerably delayed and this case is important in showing that one must avoid being unduly hasty in diagnosing irrecoverable renal damage and abandoning hope of survival.

\section{Case Reports}

Case 1. A boy with urethral valves was admitted at the age of 6 days with convulsions. His bladder and kidneys were palpably distended. The blood urea was $178 \mathrm{mg} . / 100 \mathrm{ml}$. and the urine was infected with $E$. coli. Bilateral loop ureterostomies were performed, but the child's general condition remained extremely poor. He was persistently uraemic and acidaemic and needed a very large fluid intake and urinary output to maintain him in anything approaching a reasonable clinical and biochemical state; at the age of 5 weeks this fluid exchange amounted to a daily intake of $1,300 \mathrm{ml}$. and an output of $840 \mathrm{ml}$., nearly three times the normal volumes for his size. He had repeated attacks of vomiting and diarrhoea and was quite unable to take orally the copious intake he required so that intravenous infusions were needed almost continually. At the age of 4 months his weight was still below his birth weight of $7 \frac{1}{2} \mathrm{lb}$. $(3.4 \mathrm{~kg}$.). 
Then, quite suddenly, he improved, began to gain weight and was able to maintain homeostasis by oral intake alone. His intravenous pyelogram at this time showed that, although the right ureterostomy was draining urine, the kidney did not concentrate the dye; on the left side there was hydronephrosis and hydroureter (Fig. 2). The urethral valves were resected by an open, retropubic operation, and by the age of 6 months the ureterostomies were closed and the child was micturating normally. He was last seen at the age of 18 months when his clinical condition was excellent; his blood urea was $54 \mathrm{mg}$./ $100 \mathrm{ml}$. and his urine was uninfected and free from pus.

Comment. It is likely that part of the clinical improvement that occurs in such cases is due not to the recovery of renal function itself but to the drainage of infection and the relief therefore of such general septic and toxic effects as vomiting, diarrhoea, impaired hepatic function, often with jaundice and depressed marrow activity resulting in anaemia or, as in one of my cases, in severe thrombocytopenia with haemorrhages.

Besides its benefit to the upper urinary tract, temporary ureterostomy is of value in restoring the function of a chronically distended bladder. This was illustrated by a boy with an unusual type of urethral obstruction associated with skeletal abnormalities.

Case 2. This boy presented at the age of 3 years 8 months with the history that he had been dribbling urine constantly since birth. His appearance was that of the Marfan arachnodactyly syndrome; he was tall and thin with poor musculature, a long narrow skull and long limbs and digits. There was a moderate degree of genu valgum (Fig. 3). The child's bladder was persistently distended and there was a constant urinary leak. Compression of the bladder produced a very fine jet of urine from the meatus. The nervous system and the anal sphincters were normal. On admission the blood chemistry was within normal limits and the urine was uninfected. Intravenous pyelography showed a severe degree of bilateral hydronephrosis and hydroureter (Fig. 4). The radiographs also showed a wide spinal canal in the lower lumbar region, but air myelography revealed no abnormality. Attempted instrumentation showed that the urethral meatus was normal but that the penile urethra was extremely narrow so that only a very fine polythene catheter could be passed. A cystourethrogram showed that there was a duplication of the penile part of the urethra (Fig. 5). At operation the presence of the double urethra was confirmed; the interurethral septum was excised and both urethrae were laid open from the single meatus to the bulb, as in the first stage of the Johanson operation for stricture. In spite of the fact that the proximal urethra was of adequate calibre, the operation proved to be of no benefit; the patient continued to dribble urine which was by now infected and he was pyrexial and ill. Prolonged drainage was obviously needed so a 'tubeless cystostomy'

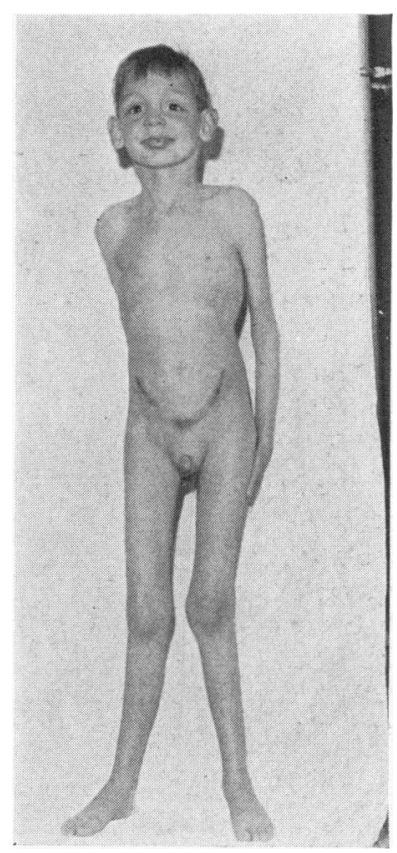

Fig. 3.-Case 2 at the age of $4 \frac{1}{2}$ years, after ureterostomy closure. Height $45 \frac{1}{2}$ in. $\left(112 \mathrm{~cm}\right.$.) $\left(3 \frac{1}{2}\right.$ in. $(7 \cdot 6 \mathrm{~cm}$.) greater than average for his age). Arachnodactyly, poor musculature and genu valgum.

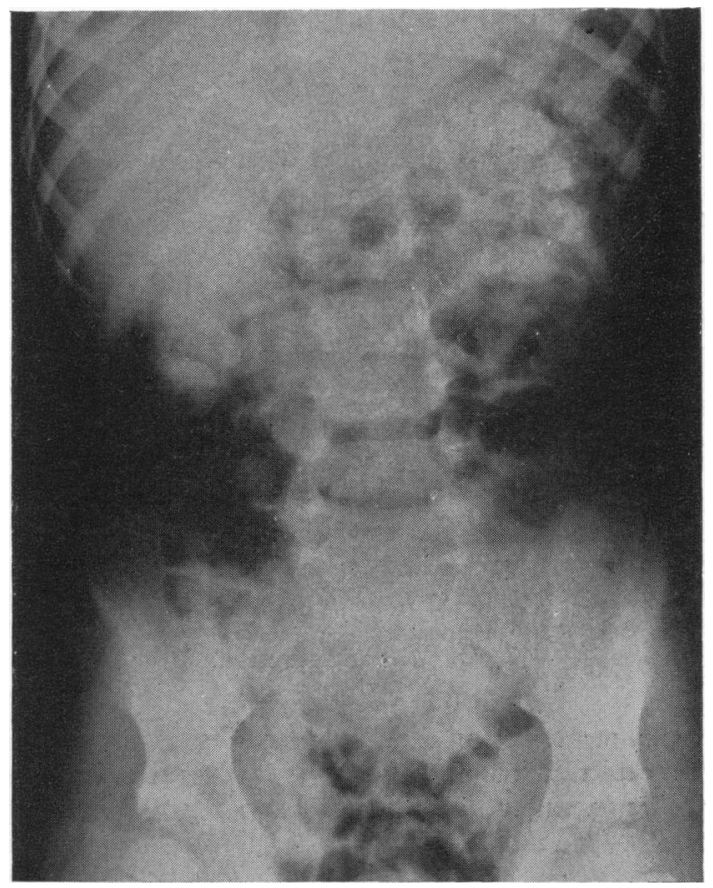

FIG. 4.-Intravenous pyelogram of Case 2 on admission. Bilateral hydronephrosis and hydroureter. Wide spinal canal in lower lumbar region shown by myelography to be without neurological signinicance. 


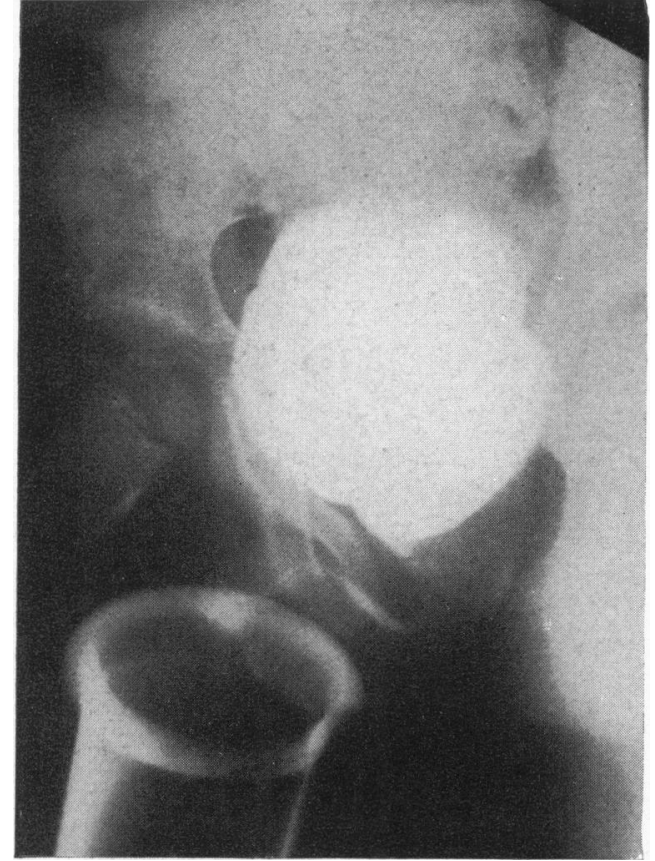

FIG. 5.-Cystourethrogram of Case 2. Obstructive duplication of penile urethra.

was carried out. It proved, however, to be extremely difficult to bring the thick-walled bladder to the surface without tension and because of subsequent retraction and stenosis of the stoma with inadequate drainage it became necessary to insert a suprapubic catheter. Repeated attempts were made to induce micturition, but when the catheter was removed the patient again dribbled from the urethra and leaked suprapubically, and his general condition became progressively worse. Fourteen weeks after his admission bilateral loop ureterostomies were performed. This led to an almost immediate clinical improvement and the child went home three weeks later wearing a collecting appliance. The ureterostomies were closed after 13 weeks when he was able to micturate quite well on demand, although he leaked urine between voidings. Gradually, his control improved so that now, 11 months after the ureterostomy closure, he is quite dry by day but often wet at night. The urine is now uninfected. He has yet to have the second stage urethral reconstruction operation.

Comment. Persistent bladder dysfunction after the removal of a congenital urinary obstruction occurs, as in the above patient, particularly in the case in which the diagnosis has been delayed. It is probably partly due to diminished vesical tone resulting from prolonged overdistension, as is seen with the atonic obstructed bladder of adults, but another, possibly more important, factor in its causation is such a child's complete lack of experience of the normal sensations associated with bladder filling and emptying so that he may need a long period of self-education of the neural arcs concerned before normal micturition and urinary control is possible. A period of ureterostomy drainage serves the dual purpose of helping to restore normal bladder activity and of protecting the upper urinary tract during the process.

In the case of acquired urinary disease the ureters do not come safely to the surface as loops and failure to diagnose what was probably an example of this rare cause of urinary obstruction in infancy led to local ureterostomy complications.

Case 3. A boy aged 6 weeks was admitted to hospital in an almost moribund state with convulsions and deep jaundice. The bladder was tensely distended above the umbilicus and both kidneys were enlarged. The blood urea was $150 \mathrm{mg} . / 100 \mathrm{ml}$. and the serum bilirubin $28 \mathrm{mg}$. $/ 100 \mathrm{ml}$. The urine obtained by catheterization was macroscopically purulent and grew $E$. coli on culture. I thought that the child must have urethral valves and decided to perform bilateral ureterostomies. Cystoscopy and cystography were omitted. When the ureters were exposed they were found to be quite dilated, but thin walled and straight. Loop ureterostomies were done, but although the ureters could be brought to the surface without tension, the mobilization led to necrosis of part of each ureter adjacent to the ureterostomy; the child's poor general condition undoubtedly contributed to the development of this complication. Later investigations showed that the renal enlargement was due to severe bilateral acute pyelonephritis with only moderate hydronephrosis and that the bladder was slightly trabeculated, with bullous cystitis, but failed to demonstrate any residual urinary obstruction whatever. A left nephrectomy was required later because of septic destruction of the kidney, but the child ultimately recovered and left hospital micturating normally. In retrospect, it appears likely that the obstruction in this case was purely inflammatory, due to a prostatitis or a basal cystitis.

\section{Discussion}

Closure of the terminal ureterostomy requires reimplantation of the ureter to the bladder and it is difficult, in my experience, to reimplant a very dilated, thick-walled ureter and avoid with certainty and permanently subsequent vesico-ureteric reflux with its consequences of persistent infection and progressive renal deterioration. The best chance of achieving a competent valve with such a ureter lies, I believe, in performing a direct anastomosis of the ureter to the bladder high on its posterior wall and supplementing the stoma with a long Bischoff 
ostioplasty. I am not, however, in a position to quote long-term results of this technique. In the case of the loop ureterostomy, the exteriorized ureter becomes oedematous and thickened, but one must resist, during the closure operation, the temptation to make things easier and neater by resecting this part of the ureter and performing an end-to-end anastomosis. One usually finds that the ureter below the ureterostomy is appreciably smaller than the portion above, and in two cases in which the ureterostomies were resected, intravenous pyelography several months after the closure operation showed that this luminal disparity persisted with, in one of them, increased dilatation of the kidney and the upper part of the ureter. In this case, where resection of the ureter was unavoidable since part of it underwent necrosis, retrograde pyelography of the sole remaining kidney six months after ureterostomy closure showed considerable delay in emptying of the ureter above the anastomosis. After one and a half hours very little of the dye injected into the renal pelvis had passed into the lower ureter and bladder (Fig. 6). Under the image intensifier one could see vigorous peristalsis to the site of the anastomosis, but no dye was seen to pass through within half an hour of the injection; one also had the impression of reversed peristalsis from this point. The delay at the anastomosis is due, I believe, not to any local narrowing, which cannot be significant with a dilated ureter, but to a failure of transmission of the peristaltic wave consequent on ureteric resection, a phenomenon that Murnaghan (1959) found to occur on occasion after complete division and reanastomosis of the ureter in the dog. This functional obstruction at the site of ureterostomy closure should be avoidable by retaining continuity of the ureteric musculature and this proved to be so in Case 2. This boy's ureterostomies were closed by mobilization of the ureter and transverse suture of the stoma, leaving the posterior wall intact. An intravenous pyelogram 10 months later showed that there was still severe dilatation of both kidneys and ureters, but no obstruction above the ureteric closures which were visible as linear-filling defects (Fig. 7).

Timing of Closure. If the child survives and thrives there must never, I believe, be any question of not closing the ureterostomies; when the patient's general condition is good, he is gaining weight and the ureterostomies are draining freely with minimal residue in the upper renal tract, the urinary obstruction can be removed if this has not already been done. The ability of the bladder to empty must

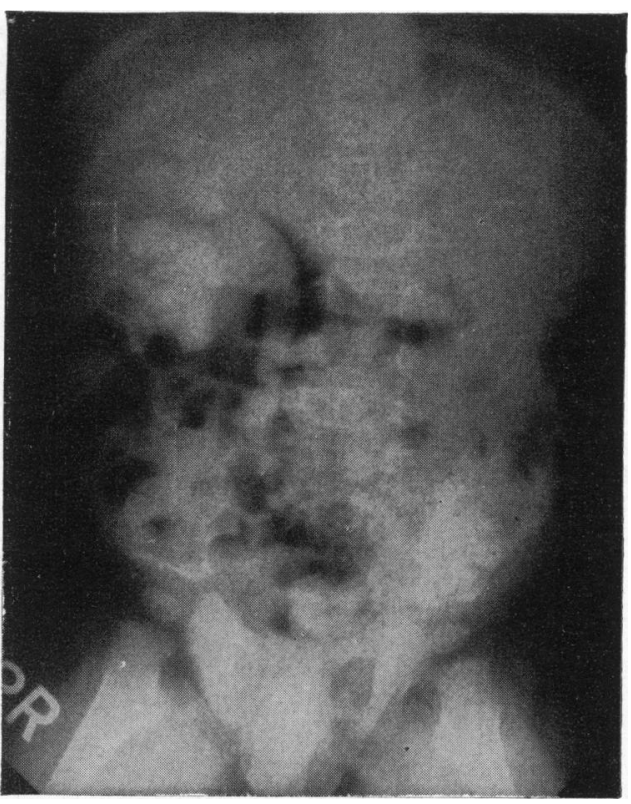

FIG. 6.-Retrograde pyelogram of Case 3, six months after closure of ureterostomy with resection of part of the ureter. Marked delay in emptying of kidney and ureter above ureteric anastomosis. Radiograph taken one and a half hours after injection of contrast medium.

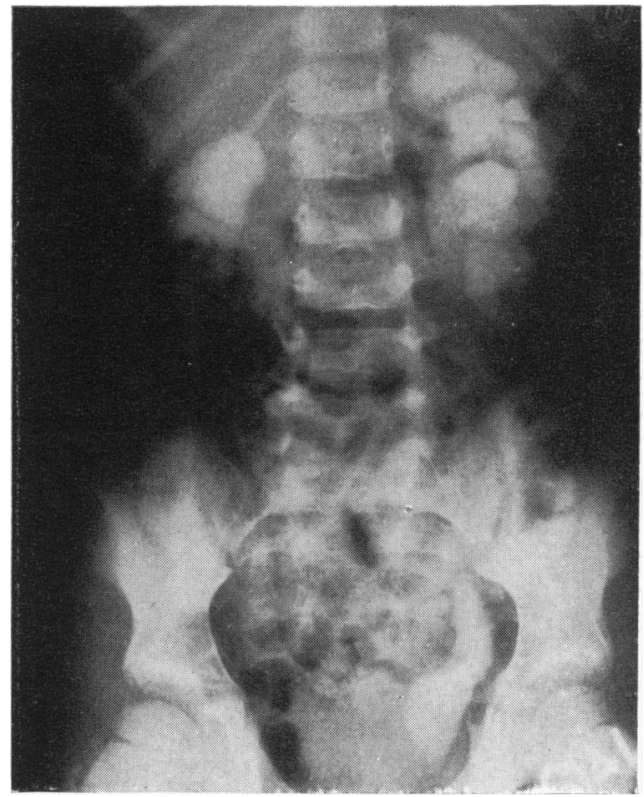

FIG. 7.-Intravenous pyelogram of Case 2, 10 months after bilateral ureterostomy closure, retaining continuity of ureteric musculature. No stasis above sites of closure, which are visible as linear filling defects. 
then be assessed, and this is particularly easy when there are loop ureterostomies; a catheter is passed into the ureter below the ureterostomy and saline is injected through it to the bladder. By connecting an intravenous drip set to the catheter the patient's capacity to micturate can be trained and observed for weeks if necessary and its adequacy confirmed radiologically. If these precautions are taken, and they are needed especially in the child with an atonic neurally dissociated bladder, one can readily judge when ureterostomy closure is safe.

The technique of temporary ureterostomy carries its own problems, although the complications I have described are undoubtedly avoidable. The method is obviously not required for every child with congenital urinary obstruction. Its great use, either as a primary elective procedure or as a method of resort when other drainage techniques have failed, is in the really ill child with a dilated, infected decompensated urinary tract which needs prolonged decompression. In such cases, and these are, of course, the patients with the most severely damaged renal tracts who are most precariously situated, I have found temporary ureterostomy a most valuable, even a life-saving, method of management.

I am indebted to Miss Isabella Forshall for allowing me to undertake radiological and biochemical investigations on two of her patients included in the series.

REFERENCES

Murnaghan, G. F. (1959). Experimental aspects of hydronephrosis. Brit. J. Urol., 31, 370.

Rickham, P. P. (1962). Advanced lower urinary obstruction in childhood. Arch. Dis. Childh., 37, 122. 